OPEN ACCESS

Edited by:

Maik Gollasch,

Charité Universitätsmedizin Berlin, Germany

Reviewed by:

Giorgos K. Sakkas,

University of Thessaly, Greece

Malcolm Koo,

Tzu Chi University of Science and

Technology, Taiwan

Abhinav Dixit,

All India Institute of Medical Sciences

Jodhpur, India

*Correspondence:

Chia-Ter Chao

b88401084@gmail.com

Specialty section:

This article was submitted to

Nephrology,

a section of the journal

Frontiers in Medicine

Received: 21 October 2021

Accepted: 14 January 2022

Published: 15 February 2022

Citation:

Chi C-Y, Lee S-Y, Chao C-T and

Huang J-W (2022) Frailty as an

Independent Risk Factor for

Depression in Patients With

End-Stage Renal Disease: A

Cross-Sectional Study.

Front. Med. 9:799544.

doi: 10.3389/fmed.2022.799544

\section{Frailty as an Independent Risk Factor for Depression in Patients With End-Stage Renal Disease: A Cross-Sectional Study}

\author{
Chun-Yi Chi ${ }^{1}$, Szu-Ying Lee ${ }^{1}$, Chia-Ter Chao ${ }^{2,3,4 *}$ and Jenq-Wen Huang ${ }^{1,4}$ \\ ${ }^{1}$ Nephrology Division, Department of Internal Medicine, National Taiwan University Hospital Yunlin Branch, Douliu, Taiwan, \\ ${ }^{2}$ Nephrology Division, Department of Internal Medicine, National Taiwan University Hospital, Taipei, Taiwan, ${ }^{3}$ Graduate \\ Institute of Toxicology, National Taiwan University College of Medicine, Taipei, Taiwan, ${ }^{4}$ Nephrology Division, Department of \\ Internal Medicine, National Taiwan University College of Medicine, Taipei, Taiwan
}

Background: Depression confers substantial disease burden globally, especially among those with chronic kidney disease (CKD). The presence of depression significantly impairs one's quality of life. Risk factors for depression in patients with CKD remain under-appreciated, and whether frailty, a geriatric phenotype, constitutes a risk factor for depression in this population is unknown.

Methods: We prospectively enrolled patients with end-stage renal disease (ESRD) undergoing hemodialysis for $>3$ months from National Taiwan University Hospital Yunlin Branch between 2019 and 2021. Clinical, physical, functional, and performance parameters were recorded, followed by frailty/sarcopenia assessment. Depression was screened for using the Geriatric Depression Scale. We analyzed the independent relationship between frailty and depression in these patients, using multiple regression analyses.

Results: Totally 151 patients with ESRD were enrolled (mean 61.1 years, 66.9\% male), among whom $16.6 \%$ had screening-identified depression. ESRD participants with depression did not differ from those without regarding most parameters except serum creatinine, functional indices, and sarcopenia/frailty status. We found that having greater frail severities was independently associated with a higher probability of depression; having FRAIL- (odds ratio [OR] 5.418) and SOF-based (OR 2.858) frailty independently correlated with a higher depression probability. A linear relation exists between a greater frail severity and the probability of depression. Using a more relaxed criterion for detecting depression, higher SOF scores remained significantly associated with an increased depression risk.

Conclusions: In patients with CKD, frailty independently correlated with a higher probability of having depression. Strategies aiming to attenuate frailty may be able to benefit those with depression simultaneously in this population.

Keywords: chronic kidney disease, depression, end-stage renal disease, frailty, geriatric phenotype, malnutrition, sarcopenia 


\section{INTRODUCTION}

Depression, characterized by an emotional turbulence presenting with somatic, cognitive, and behavioral symptoms, is one of the common psychiatric disorders that affect billions of people and confer substantial disease burden globally (1). Depression is frequently accompanied by loss of interest toward activities and relationships, and prominently impairs an individual's quality of life. Depression exhibits an increased incidence in patients with chronic kidney disease (CKD) and especially those with endstage renal disease (ESRD), up to $20 \%$ to $40 \%$ depending upon countries and assessment tools (2). Depression increases CKD patients' long-term mortality by at least $50 \%$, based on National Health and Nutrition Examination Survey (NHANES) results (3). Besides its effect on survival, depression poses a plethora of adverse influences in this population; depressed patients with CKD were found to have a higher incidence of muscle wasting and correlated with a greater degree of functional impairment, according to findings from the Dialysis Outcomes and Practice Patterns Study (DOPPS) $(4,5)$. Furthermore, depression and its predisposing traits likely modulate the incidence of CKD and its subsequent progression. A recent Mendelian randomization study showed that genetic alleles intimately associated with depressive symptoms conferred a greater risk of carrying lower estimated glomerular filtration rates (eGFRs) (6). Having depressive symptoms places patients at risk of developing accelerated renal function decline, rendering the identification of depression instrumental (7).

Risk factors for depression in patients with CKD or ESRD remain under-appreciated. Existing studies mostly involve patients with depression but without CKD; systematic reviews and meta-analyses indicated that smoking, higher body mass index (BMI), lower blood pressure, personal traits, chronic diseases, and sleep disturbance were associated with an increased risk of having depression in various populations $(8,9)$. On the other hand, these risk features may not be applicable to those with CKD. Anecdotal studies revealed that severe pain, negative illness perception, and inadequate self-esteem significantly correlated with the presence of depression in those with non-dialysis CKD (10). A longer dialysis vintage also modulated the probability of developing depression in patients with ESRD (11). Since the presence of CKD is associated with premature biological aging (12), emerging studies suggest that geriatric phenotypes demonstrate a high prevalence in this population. Frailty, in particular, is found to be highly prevalent in patients with renal insufficiency. Frailty is recently shown to correlate with the presence and severity of depression in community-dwelling older

Abbreviations: $\mathrm{BH}$, body height; $\mathrm{BMI}$, body mass index; $\mathrm{BP}$, blood pressure; BW, body weight; CHS, cardiovascular health study; CI, confidence interval; CKD, chronic kidney disease; CNAQ, Council of Nutrition Assessment Questionnaire; DOPPS, Dialysis Outcomes and Practice Patterns Study; ECOG, eastern cooperative oncology group; EFS, Edmonton frail scale; eGFR, estimated glomerular filtration rate; ESRD, end-stage renal disease; FRAIL, fatigue, resistance, ambulation, illness, and loss of weight; GDS-15, Geriatric Depression Scale-15 items; IADL, instrumental activity of daily living; MDRD, Modification of Diet in Renal Disease; NHANES, National Health and Nutrition Examination Survey; OR, odds ratio; SOF, study of osteoporotic fracture; TCS, timed chair stand; TUG, time up and go; WC, waist circumference. adults (13), but very few address the possibility whether frailty constitutes a risk factor for depression in patients with CKD. Such relationship has been hypothesized before (14) but never tested in this population. To answer this question, we used a prospectively collected cohort of patients with ESRD to analyze the connection between frailty and depression, using well-validated instruments.

\section{SUBJECTS, MATERIALS, AND METHODS Ethical Statement}

The protocol of the current project has been approved by the institutional review board of National Taiwan University Hospital (No. 201910100RINA). The details of the study protocol adhered to the Declaration of Helsinki, and all participants provided written informed consent.

\section{Recruitment of Participants and Study Procedures}

Patients with ESRD, defined as having an eGFR $<15$ $\mathrm{mL} / \mathrm{min} / 1.73 \mathrm{~m}^{2}$, undergoing hemodialysis for more than 3 months, were prospectively enrolled from the dialysis units of National Taiwan University Hospital Yunlin Branch, Douliou and Huwei branches between August 2019 and July 2021. We used the 4-variable Modification of Diet in Renal Disease (MDRD) formula for calculating eGFR. After providing informed consent, participants underwent a 3-step assessment; first, they were interviewed by dedicated nursing staff, with their demographic information (age, gender, and education level) and morbidities recorded. Second, participants underwent physical examination, with their anthropometric parameters [body weight (BW)/body height (BH), waist circumference (WC), and arm/leg circumference] and physical examination indices [blood pressure (BP), heart rate (HR), and respiratory rate] collected (15). At this stage, participants were also instructed to complete performance assessment involving upper and lower limbs, including grip strength (using a TKK dynamometer; Takei Inc., Niigata, Japan), timed chair stand (TCS), time-upand-go (TUG), and gait speed, according to protocols published previously $(16,17)$. For all performance assessment, results were obtained after averaging data from 3 repetitive tests. Finally, as the last step, dedicated staff counseled with the participants and administered self-report questionnaires including functional evaluation (eastern cooperative oncology group [ECOG], Karnofsky performance scale, Barthel index, Katz index, and Lawton-Brody instrumental activity of daily living [IADL]), sarcopenia assessment (SARC-F questionnaire), frailty status evaluation [Edmonton frail scale (EFS), Study of Osteoporotic Fractures (SOF) scale, and Fatigue, Resistance, Ambulation, Illness, Loss of weight (FRAIL) scale], and nutritional/appetite screening [Council of Nutrition Assessment Questionnaire (CNAQ)]. The validity of instruments for evaluating frailty, sarcopenia, and nutritional levels in this study has been tested and reassured in patients with CKD and ESRD based on other reports and our prior findings (17-19). Those with a SARC-F score $\geq 4$ were defined as having sarcopenia, while those with a SOF, FRAIL, or EFS score $\geq 2,3$, and 8 were considered frail, respectively, according to their original schemes. Finally, at least 
$10 \mathrm{~mL}$ of peripheral blood was obtained from participants, sent for laboratory tests including hemogram, serum biochemistry and electrolytes, and metabolic parameters (glucose, lipid profile, and uric acid).

\section{Outcome Assessment}

In this study, we screened these patients regarding whether they had depression, using the Geriatric Depression Scale-15 items (GDS-15). GDS-15 has been recommended as a useful tool to screen for depression in older adults during acute and chronic settings, and also in patients with $\operatorname{CKD}(20,21)$, with a score range between 0 and 15 . Compared with other GDS instruments with different item counts (GDS-30, GDS-5, and GDS-4), GDS-15 exhibited a better recognition accuracy compared to others, owing to its advantages of preserving core messages while optimizing the amount of item load (22). GDS-15 assesses participants' depressive symptoms, psychosocial activities, life satisfaction, etc., all of which correlate closely with each other (23). After reassuring patients' cognitive status and literacy level, participants completed the GDS-15 questionnaire, consisting of 10 and 5 positive and negative responses to the presence of depression, respectively, with or without assistance from dedicated staff. Those with a GDS-15 score $\geq 10$ were identified as potentially having depression, according to the existing literature (21-23).

\section{Statistical Analysis}

For continuous variables, we compared between groups using Student's $t$-tests (if normally distributed) or Mann-Whitney U-test (if skewed distribution). For categorical variables, we compared between groups using Chi-square tests. In all analyses, a $p$-value $<0.05$ was considered statistically significant. We used IBM SPSS Statistics for Windows, Version 19.0 (Armonk, NY; IBM Corp.) in all statistical analysis.

After completing all assessments in phases 1 and 2, we divided participants into those with and without potential depression, followed by comparing their demographic profiles, comorbidities, physical examination and anthropometric parameters, performance indicators, and laboratory findings. We further examined whether there were differences between those with and without depression, regarding their functional status, frailty, sarcopenia, and nutritional status based on relevant tools. Subsequently, we used multiple regression analysis with having depression or not as the dependent variable with stepwise backward variable selection, incorporating variables with significant differences in univariate analyses. Independent variables were expressed in odds ratios (ORs) with 95\% confidence intervals and the associated $p$-values provided. Sensitivity analyses were planned a priori, including the adjustment of variable input style (categorical vs. continuous). We also tested whether the replacement of dependent variable, depression or not based on having GDS-15 $\geq 10$, with depression or susceptibility status or not based on having GDS-15 $\geq 5$, might influence our findings.

\section{RESULTS}

During the study period, we enrolled a total of 151 patients with ESRD under chronic hemodialysis, with a mean age of 61.1 years and $66.9 \%$ male (Table 1 ). The most common comorbidity among study participants was hypertension $(80.1 \%)$, followed by diabetes mellitus (47.0\%) and peptic ulcer (30.5\%). Nearly half of these patients had chronic pain (43.7\%). Participants exhibited on average fair upper and lower limb performance, with a mean TCS, TUG and gait speed of $14.9 \mathrm{~s}, 10.7 \mathrm{~s}$, and $0.77 \mathrm{~m} / \mathrm{s}$, respectively (Table 1). Participants with ESRD were mildly anemic, but had normal electrolyte panels. Their serum uric acid $(7.8 \mathrm{mg} / \mathrm{dL})$ and triglyceride $(172.2 \mathrm{mg} / \mathrm{dL})$ levels were mildly increased, and participants had mild hyperglycemia $(118.7 \mathrm{mg} / \mathrm{dL})$.

Among all, 16.6\% participants were found to have depression after screening questionnaire use. ESRD participants with potential depression did not differ from those without regarding their demographic profiles, comorbidities, physical examination parameters, anthropometric indices, performance indicators, and most laboratory data except lower serum creatinine levels $(p=$ 0.02) among the former group (Table 1).

\section{Functional Evaluation and Frailty/Sarcopenia Assessment for Study Participants}

During functional assessment, participants with ESRD were found to have minor impairment in their activity of daily living, with an average ECOG, Karnofsky performance indicators, and Barthel index scores of 0.97 out of $4,82.8$ out of 100 , and 91.3 out of 100 , respectively (Table 2 ). Approximately $17.2 \%$ participants had sarcopenia, while $12.6,19.2$, and $23.8 \%$ of them had FRAIL-, EFS-, and SOF-defined frailty, respectively. Participants with depression had significantly higher ECOG $(p=0.028)$ and instrumental activity of daily living scores $(p=$ $0.034)$ but lower Karnofsky performance indicators $(p=0.006)$ (Table 2). Those with depression were significantly more likely to have sarcopenia than those without $(p=0.032)$. Similarly, those with depression had a significantly higher prevalence of frailty (40-52\%) compared to those without (7.1-18.3\%) (Table 2). Participants without depression had better appetite in the form of higher CNAQ scores than those without $(p=0.033)$.

\section{Independent Predictors of Depression in Patients With End-Stage Renal Disease}

We then conducted multiple regression analyses to uncover independent factors associated with having depression after the screening test in study participants. After accounting for variables with significant between-group differences in univariate analysis (Tables 1, 2), including serum creatinine, functional evaluation results (Karnofsky and IADL scores), SARC-F scores, frailty scores (EFS, FRAIL, and SOF scales), and CNAQ scores, we found that having a greater frail severity, including higher EFS (OR 1.365, 95\% CI 1.057-1.762) and SOF scores (OR 3.076, 95\% CI 1.458-6.493), was independently associated with a higher risk of developing potential depression (Table 3). Sensitivity analyses were done using having frailty or not based on EFS, 
TABLE 1 | Baseline characteristics of patients with end-stage renal disease enrolled in this study.

\begin{tabular}{|c|c|c|c|c|}
\hline & $\begin{array}{c}\text { Total } \\
(n=151)\end{array}$ & $\begin{array}{c}\text { Without } \\
\text { depression } \\
(n=126)\end{array}$ & $\begin{array}{c}\text { With } \\
\text { depression } \\
(n=25)\end{array}$ & $\begin{array}{c}p- \\
\text { value }\end{array}$ \\
\hline \multicolumn{5}{|l|}{ Basic data } \\
\hline Age (years) & $61.1 \pm 12.0$ & $61.0 \pm 11.4$ & $61.5 \pm 14.7$ & 0.863 \\
\hline Sex (male \%) & $101(66.9)$ & $86(68.3)$ & $15(60.0)$ & 0.426 \\
\hline Education & & & & 0.284 \\
\hline None & $16(10.6)$ & $12(9.5)$ & $4(16.0)$ & \\
\hline Elementary school & $32(21.2)$ & $24(19.0)$ & $8(32.0)$ & \\
\hline High school & $84(55.6)$ & $74(58.7)$ & $10(40.0)$ & \\
\hline College or higher & 19 (12.6) & $16(12.7)$ & $3(12.0)$ & \\
\hline \multicolumn{5}{|l|}{ Comorbidity } \\
\hline Diabetes mellitus (\%) & $71(47.0)$ & $58(46.0)$ & $13(52.0)$ & 0.588 \\
\hline Hypertension (\%) & $121(80.1)$ & $102(81.0)$ & $19(76.0)$ & 0.574 \\
\hline Cirrhosis (\%) & $6(4.0)$ & $5(4.0)$ & $1(4.0)$ & 0.994 \\
\hline Coronary artery disease (\%) & $32(21.2)$ & $25(19.8)$ & $7(28.0)$ & 0.365 \\
\hline $\begin{array}{l}\text { Acute myocardial infarction } \\
(\%)\end{array}$ & $8(5.3)$ & $6(4.8)$ & $2(8.0)$ & 0.512 \\
\hline Heart failure (\%) & $34(22.5)$ & $26(20.6)$ & $8(32.0)$ & 0.217 \\
\hline $\begin{array}{l}\text { Peripheral vascular } \\
\text { disease (\%) }\end{array}$ & $6(4.0)$ & $4(3.2)$ & $2(8.0)$ & 0.262 \\
\hline Atrial fibrillation (\%) & $1(0.7)$ & $1(0.8)$ & $0(0)$ & 0.658 \\
\hline COPD (\%) & $6(4.0)$ & $5(4.0)$ & $1(4.0)$ & 0.994 \\
\hline Rheumatology disorders (\%) & $5(3.3)$ & $4(3.2)$ & $1(4.0)$ & 0.834 \\
\hline Malignancy (\%) & $17(11.3)$ & $13(10.3)$ & $4(16.0)$ & 0.415 \\
\hline Peptic ulcer (\%) & $46(30.5)$ & $37(29.4)$ & $9(36.0)$ & 0.513 \\
\hline $\begin{array}{l}\text { Prior cerebrovascular } \\
\text { accident (\%) }\end{array}$ & $9(6.0)$ & $7(5.6)$ & $2(8.0)$ & 0.640 \\
\hline Hemiplegia (\%) & $2(1.3)$ & $2(1.6)$ & $0(0)$ & 0.529 \\
\hline Chronic pain (\%) & $66(43.7)$ & $52(41.3)$ & $14(56.0)$ & 0.177 \\
\hline \multicolumn{5}{|l|}{ Physical examination } \\
\hline $\begin{array}{l}\text { Blood pressure-systolic } \\
(\mathrm{mmHg})\end{array}$ & $147.0 \pm 28.9$ & $147.7 \pm 29.1$ & $143.6 \pm 28.7$ & 0.516 \\
\hline $\begin{array}{l}\text { Blood pressure-diastolic } \\
(\mathrm{mmHg})\end{array}$ & $71.0 \pm 13.6$ & $71.8 \pm 14.0$ & $67.0 \pm 10.9$ & 0.108 \\
\hline Heart rate (per min) & $75.8 \pm 11.8$ & $75.8 \pm 11.6$ & $75.6 \pm 13.1$ & 0.943 \\
\hline Respiratory rate (per min) & $16.8 \pm 1.8$ & $16.8 \pm 1.8$ & $16.6 \pm 1.5$ & 0.613 \\
\hline \multicolumn{5}{|c|}{ Anthropometric parameters } \\
\hline Body weight (kg) & $63.8 \pm 15.1$ & $63.1 \pm 14.5$ & $67.1 \pm 18.0$ & 0.226 \\
\hline Body height $(\mathrm{cm})$ & $162.9 \pm 8.3$ & $162.7 \pm 8.2$ & $163.5 \pm 8.9$ & 0.662 \\
\hline Body mass index $\left(\mathrm{kg} / \mathrm{m}^{2}\right)$ & $23.9 \pm 4.3$ & $23.7 \pm 4.2$ & $24.8 \pm 5.0$ & 0.215 \\
\hline Waist circumference (cm) & $86.8 \pm 12.9$ & $86.8 \pm 13.0$ & $87.1 \pm 12.8$ & 0.906 \\
\hline Mid-arm circumference (cm) & $26.9 \pm 4.1$ & $26.9 \pm 3.9$ & $26.9 \pm 4.9$ & 0.992 \\
\hline Mid-leg circumference (cm) & $31.9 \pm 4.0$ & $31.9 \pm 3.8$ & $32.0 \pm 5.1$ & 0.913 \\
\hline \multicolumn{5}{|l|}{ Performance indicators } \\
\hline Grip strength (lb) & $155.7 \pm 66.6$ & $159.3 \pm 63.5$ & $137.5 \pm 79.3$ & 0.136 \\
\hline Timed chair stand (s) & $14.9 \pm 9.8$ & $14.8 \pm 10.4$ & $15.1 \pm 5.3$ & 0.892 \\
\hline Timed up and go (s) & $10.7 \pm 3.4$ & $10.5 \pm 3.0$ & $11.4 \pm 5.0$ & 0.243 \\
\hline Gait speed (m/s) & $0.77 \pm 0.15$ & $0.77 \pm 0.15$ & $0.77 \pm 0.13$ & 0.918 \\
\hline \multicolumn{5}{|l|}{ Laboratory profile } \\
\hline \multicolumn{5}{|l|}{ Hemogram } \\
\hline Leukocyte $(\mathrm{K} / \mu \mathrm{L})$ & $7.1 \pm 7.6$ & $7.2 \pm 8.3$ & $6.5 \pm 2.3$ & 0.689 \\
\hline Hemoglobin (g/dL) & $10.5 \pm 1.5$ & $10.5 \pm 1.4$ & $10.8 \pm 1.9$ & 0.273 \\
\hline
\end{tabular}

(Continued)
TABLE 1 | Continued

\begin{tabular}{lcccc}
\hline & $\begin{array}{c}\text { Total } \\
(\boldsymbol{n}=\mathbf{1 5 1})\end{array}$ & $\begin{array}{c}\text { Without } \\
\text { depression } \\
(\boldsymbol{n}=\mathbf{1 2 6})\end{array}$ & $\begin{array}{c}\text { With } \\
\text { depression } \\
(\boldsymbol{n}=\mathbf{2 5})\end{array}$ & $\begin{array}{c}\boldsymbol{p} \text { - } \\
\text { value }\end{array}$ \\
\hline Platelet (K/ $\mathrm{LL})$ & $169.5 \pm 54.1$ & $167.4 \pm 50.6$ & $179.6 \pm 69.6$ & 0.305 \\
Biochemistry & & & & \\
Urea nitrogen (mg/dL) & $85.1 \pm 19.8$ & $84.5 \pm 20.3$ & $87.8 \pm 17.7$ & 0.445 \\
Creatinine (mg/dL) & $12.3 \pm 2.4$ & $12.5 \pm 2.2$ & $11.3 \pm 2.7$ & 0.020 \\
Albumin (mg/dL) & $4.0 \pm 0.3$ & $4.0 \pm 0.3$ & $4.0 \pm 0.3$ & 0.898 \\
Sodium (meq/L) & $136.3 \pm 3.0$ & $136.4 \pm 2.9$ & $135.6 \pm 3.7$ & 0.202 \\
Potassium (meg/L) & $4.7 \pm 0.7$ & $4.7 \pm 0.7$ & $4.8 \pm 0.7$ & 0.435 \\
Calcium (mmol/L) & $2.4 \pm 0.2$ & $2.4 \pm 0.2$ & $2.4 \pm 0.2$ & 0.252 \\
Phosphate (mg/dL) & $5.2 \pm 1.6$ & $5.2 \pm 1.7$ & $5.1 \pm 1.4$ & 0.854 \\
Metabolic & & & & \\
Uric acid (mg/dL) & $7.8 \pm 1.8$ & $7.8 \pm 1.8$ & $7.6 \pm 2.1$ & 0.622 \\
Total cholesterol (mg/dL) & $151.9 \pm 40.7$ & $151.0 \pm 38.6$ & $156.4 \pm 50.4$ & 0.543 \\
Triglyceride (mg/dL) & $172.2 \pm$ & $171.3 \pm$ & $177.2 \pm$ & 0.845 \\
& 138.0 & 143.7 & 106.5 & \\
Low density lipoprotein & $78.8 \pm 29.9$ & $78.2 \pm 29.0$ & $82.0 \pm 34.8$ & 0.569 \\
cholesterol (mg/dL) & & & & \\
Fasting glucose (mg/dL) & $118.7 \pm 51.8$ & $116.1 \pm 47.5$ & $131.8 \pm 69.2$ & 0.166 \\
\hline
\end{tabular}

COPD, chronic obstructive pulmonary disease.

FRAIL, and SOF scales replacing frailty-assessing scores; we similarly revealed that having FRAIL- (OR 5.418) and SOFbased (OR 2.858) frailty independently correlated with a higher depression probability (Table 3). Alternatively, we used a more relaxed criterion, having a GDS $\geq 5$, as the dependent variable in another set of regression analysis; we discovered that higher SOF scores remained significantly associated with an increased risk (Table 3).

Based on our results that the prevalence of potential depression in those without and with SOF-defined frailty was $10.4 \%$ and $36.1 \%$, respectively, and that the alpha value was set at 0.05 , we could derive a post-hoc power of $91.8 \%$ for detecting difference of a dichotomous endpoint.

\section{DISCUSSION}

In this study, we prospectively enrolled a group of patients with ESRD and comprehensively assessed their baseline clinical, physical, functional, and performance status, followed by depression screening. After adjusting for potential confounders, we were able to show that frailty was an independent factor associated with having depression in these patients, in a graded fashion. This phenomenon serves to remind physicians that frailty evaluation may partially assist them in determining the probability of depression among patients with CKD, and that frailty-curbing strategy may potentially benefit CKD patients with depression as well.

The prevalence of depression ranged between 16.6 and 18.5\% in the current study. Compared to results reported by others (25 to 35\%) (2), the prevalence of depression was modestly lower; several reasons might be responsible for this phenomenon. First of all, the nutritional status of our study participants 
TABLE 2 | Functional and geriatric syndrome evaluation results of study participants.

\begin{tabular}{|c|c|c|c|c|}
\hline & $\begin{array}{c}\text { Total } \\
(n=151)\end{array}$ & $\begin{array}{l}\text { Without } \\
\text { depression } \\
(n=126)\end{array}$ & $\begin{array}{c}\text { With } \\
\text { depression } \\
(n=25)\end{array}$ & $\begin{array}{c}p- \\
\text { value }\end{array}$ \\
\hline \multicolumn{5}{|l|}{ Functional evaluation } \\
\hline ECOG & $0.97 \pm 0.77$ & $0.91 \pm 0.73$ & $1.28 \pm 0.89$ & 0.028 \\
\hline $\begin{array}{l}\text { Karnofsky performance } \\
\text { indicators }\end{array}$ & $82.8 \pm 14.6$ & $84.2 \pm 13.7$ & $75.6 \pm 16.9$ & 0.006 \\
\hline Barthel index scores & $91.3 \pm 22.2$ & $92.8 \pm 20.8$ & $83.6 \pm 27.6$ & 0.058 \\
\hline Katz index scores & $5.4 \pm 1.6$ & $5.5 \pm 1.5$ & $4.9 \pm 2.1$ & 0.083 \\
\hline Lawton-Brody IADL scores & $1.7 \pm 2.3$ & $1.6 \pm 2.2$ & $2.6 \pm 2.6$ & 0.034 \\
\hline \multicolumn{5}{|l|}{ Sarcopenia } \\
\hline SARC-F scores & $1.6 \pm 2.5$ & $1.4 \pm 2.4$ & $2.9 \pm 3.0$ & 0.006 \\
\hline Sarcopenia & $26(17.2)$ & $18(14.3)$ & $8(32.0)$ & 0.032 \\
\hline \multicolumn{5}{|l|}{ Frailty } \\
\hline Edmonton frail scale scores & $5.1 \pm 2.6$ & $4.8 \pm 2.4$ & $6.8 \pm 2.8$ & $<0.001$ \\
\hline EFS-defined frailty (\%) & $29(19.2)$ & $19(15.1)$ & $10(40.0)$ & 0.004 \\
\hline FRAIL scale scores & $0.90 \pm 1.26$ & $0.75 \pm 1.10$ & $1.68 \pm 1.68$ & 0.001 \\
\hline FRAIL-defined frailty (\%) & 19 (12.6) & $9(7.1)$ & $10(40.0)$ & $<0.001$ \\
\hline SOF scale scores & $1.00 \pm 0.76$ & $0.90 \pm 0.73$ & $1.56 \pm 0.71$ & $<0.001$ \\
\hline SOF-defined frailty (\%) & $36(23.8)$ & $23(18.3)$ & $13(52.0)$ & $<0.001$ \\
\hline \multicolumn{5}{|l|}{ Nutritional evaluation } \\
\hline CNAQ scores & $26.9 \pm 3.1$ & $27.1 \pm 2.9$ & $25.7 \pm 3.8$ & 0.033 \\
\hline
\end{tabular}

CNAQ, Council on Nutrition Appetite Questionnaire; ECOG, Eastern Cooperative Oncology Group; EFS, Edmonton frail scale; IADL, instrumental activity of daily living; SOF, Study of Osteoporotic Fracture.

TABLE $\mathbf{3}$ | Independent factors associated with having depression among patients with end-stage renal disease.

\begin{tabular}{|c|c|c|c|}
\hline Variables $^{\&}$ & Odds ratio & $95 \%$ confidence interval & $P$-value \\
\hline \multicolumn{4}{|c|}{ Having depression, incorporating frailty scores } \\
\hline EFS scores (per 1 score) & 1.365 & $1.057-1.762$ & 0.017 \\
\hline SOF scores (per 1 score) & 3.076 & $1.458-6.493$ & 0.003 \\
\hline \multicolumn{4}{|c|}{ Having depression, incorporating frailty status } \\
\hline FRAIL-based frailty & 5.418 & $1.723-17.032$ & 0.004 \\
\hline SOF-based frailty & 2.858 & $1.032-7.914$ & 0.043 \\
\hline \multicolumn{4}{|c|}{ Having depression or depression susceptibility } \\
\hline SOF scores (per 1 score) & 3.517 & $1.642-7.532$ & 0.001 \\
\hline
\end{tabular}

appeared fair, with relatively good muscle power and functional status. This assertion is supported by their average BMI (23.9 $\pm 4.3 \mathrm{~kg} / \mathrm{m}^{2}$ ) and fair gait speed/grip strength (Table 1) relative to the mean values obtained previously in Taiwanese patients with ESRD (16). On the other hand, the sensitivity of our depression screening instrument may need to be optimized. There are other ways of detecting depression in patients with CKD, including Beck depression inventory, Hamilton rating scale, major depression inventory, center for epidemiological studies depression screening index, etc. (2), but heterogeneity in results is not uncommon. Specifically, it is speculated that the estimation of depression prevalence may be lower when patients are assessed by clinical interview compared to data obtained by self-report (2). Since our participants were assessed by a hybrid of self-report and clinical interview, it is likely that the prevalence estimate could be somewhat lower. Nonetheless, the relationship between depression identified by different instruments and adverse outcomes remains consistent across tools.

The paths connecting frailty to the inception of depression, though frequently under-recognized, can be complex. Frailty has been proposed to be conductive to having a mindset of suboptimal health perception and inadequate competence in self-care (24); possessing illness perceptions including a greater symptomatology, less personal control, and maladaptive coping strategies has been shown to increase the distress level of patients with CKD (25), predisposing them to the subsequent development of depression. Patients with frailty frequently report the co-presence of other geriatric syndromes such as malnutrition, polypharmacy, and functional impairment. Geriatric phenotypes, including malnutrition and polypharmacy, has been suggested to independently correlate with reporting depressive symptoms (26), serving as another rationale for linking frailty to depression. Alternatively, frailty may co-exist with a greater severity of occult inflammation; a meta-analysis showed that frail patients had significantly higher circulating levels of C-reactive protein and interleukin-6 than non-frail ones (27). Chronic inflammation, or the ingestion of a proinflammatory diet, potentially increases the risk of depression (28), constituting another link between frailty and depression. The strength of such link may become more prominent in patients with CKD, whose severity of inflammation outnumbers those without (29). From these arguments, we can presume that frailty may increase the risk of depression in patients with CKD, through multiple mechanisms.

We showed that results generated from one of the three frailty-assessing instruments (SOF scale) exhibited a consistent association with the risk of depression across different models, while the other two (EFS and FRAIL scale) were conditionally associated (Table 3). There are differences regarding the scale components, the predictive accuracy, the ease of administration, and the applicability between the 3 instruments. SOF scale has fewer items, is easier for use, and has been widely validated in various populations for outcome prediction, but it tends to over-screen frailty (30). With these features in mind, it is expectable that SOF may potentially be more sensitive for identifying those with earlier presentations of frailty compared to other instruments; indeed, we found that SOF identified a significantly higher proportion of patients with frailty in our cohort. Prior studies revealed that in certain population, SOF scale exhibited better detection ability for adverse outcomes compared to Cardiovascular Health Study (CHS) scale (31). Therefore, our findings may be reasonable in light of the inherent differences between frailty-detecting instruments.

Existing interventions to ameliorate depressive symptoms in patients with CKD include wellbeing enhancement through counseling or electronic apps (32), exercise regimens such as cycling, strengthening, pilates, jogging or home-based ones 
(33), mind-body interventions such as yoga or relaxation therapies (34), pharmaceutical options (specific serotoninselective reuptake inhibitor) (35). However, available options more or less have their disadvantages; patients with CKD already have high pill burden and multimorbidity, which renders them reluctant to receive pharmacological treatments or predisposes them to side effects. Psychiatric services, a quintessential part of depression management, may be unavailable due to staff shortage or uneven distributions, especially for those who do not live in urban areas. It would benefit patients with CKD if more treatment options can be tested for the management of depression in this population. Based on our findings, we propose that frailty-targeted interventions may an alternative choice if we aim to reduce the probability of depression in these patients. For example, dedicated exercise training, comorbidity management, senolytics, etc. may all be potential options for antifrailty purpose (36). It would be tempting to pursue these options as adjunct options for counteracting depression in patients with $\mathrm{CKD}$, although more studies are needed in this regard.

Our study has its strengths and limitations. In our study, we collected a comprehensive set of variables, ranging from demographic, morbidities, anthropometric, physical, functional, and laboratory parameters, as well as frailty, nutrition, and sarcopenia assessment results. This approach likely reduced the probability of result influenced by most residual confounding factors. We used multiple frailty-assessing instruments to evaluate frailty, and the results were robust. However, limitations do exist. Our sample size was not large, and statistical efficiency might not be sufficient. In addition, as discussed above, the sensitivity of our depression-assessing instrument might vary according to the methods of administration and possibly patient features. For confirming the diagnosis of depression, a psychiatrist evaluation and criteria fulfillment would be needed, but such service could be time-consuming and not readily available anytime. There are opinions suggesting that the utilization of depression screening tests may assist in earlier detection and potentially outcome improvement (37). Therefore, we used the widely applicable GDS to screen for depression in our patients. Our study is cross-sectional in nature, so a causal relationship cannot be ascertained between frailty and depression in these patients. There are theories and investigations showing that depression may also increase the risk of frailty (38), suggesting that a bi-directional relationship potentially exists between frailty and depression. Nonetheless, we could not derive such conclusion based on our results. Finally, our patients with ESRD were of Asian ethnicity and received chronic hemodialysis only, like in our prior experimental and clinical work $(39,40)$, and extrapolation of our findings to those of other ethnicities or under chronic peritoneal dialysis

\section{REFERENCES}

1. GBD 2019 Diseases and injuries Collaborators. Global burden of 369 diseases and. injuries in 204 countries and territories, 1990-2019: a systematic analysis for the Global Burden of Disease Study 2019. Lancet. (2020) 396:120422. doi: $10.1016 /$ S0140-6736(20)30925-9 would not be feasible. Broader inclusion criteria and the involvement of a cohort follow-up design would better answer these questions.

\section{CONCLUSION}

We prospectively included a cohort of patients with ESRD under chronic hemodialysis and documented their baseline status of depression, using a validated instrument, along with an extensive array of interfering variables. After adjustment, we discovered that the presence of frailty was independently associated with a higher risk of exhibiting depression, while a greater frail severity correlated with an increased risk as well. Although a definitive conclusion cannot be obtained based on the current findings, we believe that the link between frailty and depression truly exists among patients with ESRD, and that strategies aiming to attenuate frailty may be able to benefit those with depression simultaneously.

\section{ETHICS STATEMENT}

The studies involving human participants were reviewed and approved by the Institutional Review Board of the National Taiwan University Hospital (No. 201910100RINA). The patients/participants provided their written informed consent to participate in this study.

\section{AUTHOR CONTRIBUTIONS}

C-TC and J-WH: study design. S-YL, C-TC, and J-WH: data analysis. C-YC, SY-L, C-TC, and J-WH: article drafting. All authors approved the final version of the manuscript.

\section{FUNDING}

The study was financially sponsored by National Taiwan University Hospital Yunlin Branch (NTUHYL 110.N004) and Ministry of Science and Technology, Taiwan (MOST 109-2314B-002-193-MY3 and MOST 108-2314-B-002-062-MY3). The sponsors have no role in the study design, data collection, analysis, and result interpretation of this study.

\section{ACKNOWLEDGMENTS}

We are grateful to the Second Core Laboratory, Department of Medical Research of National Taiwan University Hospital and the Genomic Research Center of National Taiwan University College of Medicine for their technical input.

2. Palmer S, Vecchio M, Craig JC, Tonelli M, Johnson DW, Nicolucci A Prevalence of depression in chronic kidney disease: systematic review and meta-analysis of observational studies. Kidney Int. (2013) 84:17991. doi: 10.1038/ki.2013.77

3. Ozieh MN, Garacci E, Walker RJ, Palatnik A, Egede LE. The cumulative impact of social determinants of health factors on mortality in adults 
with diabetes and chronic kidney disease. BMC Nephrol. (2021) 22:76. doi: 10.1186/s12882-021-02277-2

4. Kurita N, Wakita T, Fujimoto S, Yanagi M, Koitabashi K, Suzuki T. Hopelessness and depression predict sarcopenia in advanced CKD and dialysis: a multicenter cohort study. J Nutr Health Aging. (2021) 25:5939. doi: 10.1007/s12603-020-1556-4

5. Brown EA, Zhao J, McCullough K, Fuller DS, Figueiredo AE, Bieber B, et al. Burden of kidney disease, health-related quality of life, and employment among patients receiving peritoneal dialysis and in-center hemodialysis: findings from the DOPPS program. Am J Kidney Dis. (2021) 78:489500.e1. doi: 10.1053/j.ajkd.2021.02.327

6. Park S, Lee S, Kim Y, Lee Y, Kang MW, Kim K. Causal effects of positive affect, life satisfaction, depressive symptoms, and neuroticism on kidney function: a mendelian randomization study. J Am Soc Nephrol. (2021) 32:1484-96. doi: 10.1681/ASN.2020071086

7. Zhang Z, He P, Liu M, Zhou C, Liu C, Li H. Association of depressive symptoms with rapid kidney function decline in adults with normal kidney function. Clin J Am Soc Nephrol. (2021) 16:889-97. doi: 10.2215/CJN.18441120

8. Chaplin AB, Daniels NF, Ples D, Anderson RZ, Gregory-Jones A, Jones PB. Longitudinal association between cardiovascular risk factors and depression in young people: a systematic review and meta-analysis of cohort studies. Psychol Med. (2021) 1-11. doi: 10.1017/S0033291721002488

9. Maier A, Riedel-Heller SG, Pabst A, Luppa M. Risk factors and protective factors of depression in older people 65+. A systematic review PLOS ONE. (2021) 16:e251326. doi: 10.1371/journal.pone.0251326

10. Duan D, Yang L, Zhang $M$, Song $X$, Ren W. Depression and associated factors in chinese patients with chronic kidney disease without dialysis: a cross-sectional study. Front Public Health. (2021) 9:605651. doi: 10.3389/fpubh.2021.605651

11. Elkheir HK, Wagaella AS, Badi S, Khalil A, Elzubair TH, Khalil A. Prevalence and risk factors of depressive symptoms among dialysis patients with endstage renal disease (ESRD) in Khartoum, Sudan: a cross-sectional study. $J$ Family Med Prim Care. (2020) 9:3639-43. doi: 10.4103/jfmpc.jfmpc_1229_19

12. Kooman JP, Kotanko P, Schols AM, Shiels PG, Stenvinkel P. Chronic kidney disease and premature ageing. Nat Rev Nephrol. (2014) 10:73242. doi: 10.1038/nrneph.2014.185

13. Hayajneh AA, Hammouri H, Rababa M, Al-Rawashedeh S, Wallace DC, Alsatari ES. frailty and its correlates in cognitively intact communitydwelling older adults. Dement Geriatr Cogn Disord. (2021) 50:35763. doi: $10.1159 / 000519054$

14. Wu PY, Chao CT, Chan DC, Huang JW, Hung KY. Contributors, risk associates, and complications of frailty in patients with chronic kidney disease: a scoping review. Ther Adv Chronic Dis. (2019) 10:2040622319880382. doi: 10.1177/2040622319880382

15. Chao CT, Han DS, Huang JW. Circulating microRNA-125b levels are associated with the risk of vascular calcification in healthy community-dwelling older adults. Front Cardiovasc Med. (2021) 8:624313. doi: $10.3389 / \mathrm{fcvm} .2021 .624313$

16. Chen SI, Chiang CL, Chao CT, Chiang CK, Huang JW. Gustatory dysfunction is closely associated with frailty in patients with chronic kidney disease. J Ren Nutr. (2021) 31:49-56. doi: 10.1053/j.jrn.2020.06.006

17. Sung CC, Liao MT, Chao CT. Independent determinants of appetite impairment among patients with stage 3 or higher chronic kidney disease: a prospective study. Nutrients. (2021) 13:2863. doi: 10.3390/nu13082863

18. Chao CT, Hsu YH, Chang PY, He YT, Ueng RS, Lai CF. Simple self-report FRAIL scale might be more closely associated with dialysis complications than other frailty screening instruments in rural chronic dialysis patients. Nephrology. (2015) 20:321-8. doi: 10.1111/nep.12401

19. Chao CT, Wang J, Chien KL. Both pre-frailty and frailty increase healthcare utilization and adverse health outcomes in patients with type 2 diabetes mellitus. Cardiovasc Diabetol. (2018) 17:130. doi: 10.1186/s12933-018-0772-2

20. Dennis M, Kadri A, Coffey J. Depression in older people in the general hospital: a systematic review of screening instruments. Age Ageing. (2012) 41:148-54. doi: 10.1093/ageing/afr169
21. Kondo K, Antick JR, Ayers CK, Kansagara D, Chopra P. Depression screening tools for patients with kidney failure: a systematic review. Clin J Am Soc Nephrol. (2020) 15:1785-95. doi: 10.2215/CJN.05540420

22. Krishnamoorthy Y, Rajaa S, Rehman T. Diagnostic accuracy of various forms of geriatric depression scale for screening of depression among older adults: systematic review and meta-analysis. Arch Gerontol Geriatr. (2020) 87:104002. doi: 10.1016/j.archger.2019.104002

23. Balsamo M, Cataldi F, Carlucci L, Padulo C, Fairfield B. Assessment of latelife depression via self-report measures: a review. Clin Interv Aging. (2018) 13:2021-44. doi: 10.2147/CIA.S114100

24. Pickard S. Health, illness and frailty in old age: a phenomenological exploration. J Aging Stud. (2018) 47:24-31. doi: 10.1016/j.jaging.2018.10.002

25. Muscat P, Weinman J, Farrugia E, Callus R, Chilcot J. Illness perceptions predict distress in patients with chronic kidney disease. BMC Psychol. (2021) 9:75. doi: 10.1186/s40359-021-00572-Z

26. Chrzastek Z, Guligowska A, Soltysik B, Pigłowska M, Borowiak E, Kostka J. Association of lower nutritional status and education level with the severity of depression symptoms in older adults-a cross sectional survey. Nutrients. (2021) 13:515. doi: 10.3390/nu13020515

27. Marcos-Pérez D, Sánchez-Flores M, Proietti S, Bonassi S, Costa S, Teixeira JP. Association of inflammatory mediators with frailty status in older adults: results from a systematic review and meta-analysis. Geroscience. (2020) 42:1451-73. doi: 10.1007/s11357-020-00247-4

28. Tolkien K, Bradburn S, Murgatroyd C. An anti-inflammatory diet as a potential intervention for depressive disorders: a systematic review and metaanalysis. Clin Nutr. (2019) 38:2045-52. doi: 10.1016/j.clnu.2018.11.007

29. Chao CT, Lin SH. Uremic toxins and frailty in patients with chronic kidney disease: a molecular insight. Int J Mol Sci. (2021) 22:6270. doi: 10.3390/ijms 22126270

30. Dent E, Kowal P, Hoogendijk EO. Frailty measurement in research and clinical practice: a review. Eur J Intern Med. (2016) 31:3-10. doi: 10.1016/j.ejim.2016.03.007

31. Henry L, Halpin L, Barnett SD, Pritchard G, Sarin E, Speir AM. Frailty in the cardiac surgical patient: comparison of frailty tools and associated outcomes. Ann Thorac Surg. (2019) 108:16-22. doi: 10.1016/j.athoracsur.2019.03.009

32. Dingwall KM, Sweet M, Cass A, Hughes JT, Kavanagh D, Howard K. Effectiveness of wellbeing intervention for chronic kidney disease (WICKD): results of a randomised controlled trial. BMC Nephrol. (2021) 22:136. doi: 10.1186/s12882-021-02344-8

33. Ferreira TL, Ribeiro HS, Ribeiro ALA, Bonini-Rocha AC, Lucena JMS, de Oliveira PA, et al. Exercise interventions improve depression and anxiety in chronic kidney disease patients: a systematic review and meta-analysis. Int Urol Nephrol. (2021) 53:925-33. doi: 10.1007/s11255-020-02612-w

34. Chu SWF, Yeam CT, Low LL, Tay WY, Foo WYM, Seng JJB. The role of mind-body interventions in pre-dialysis chronic kidney disease and dialysis patients - a systematic review of literature. Complement Ther Med. (2021) 57:102652. doi: 10.1016/j.ctim.2020.102652

35. Gregg LP, Hedayati SS. Pharmacologic and psychological interventions for depression treatment in patients with kidney disease. Curr Opin Nephrol Hypertens. (2020) 29:457-64. doi: 10.1097/MNH.0000000000000629

36. Lorenz EC, Kennedy CC, Rule AD, LeBrasseur NK, Kirkland JL, Hickson LJ. Frailty in CKD and transplantation. Kidney Int Rep. (2021) 6:227080. doi: 10.1016/j.ekir.2021.05.025

37. Costantini L, Costanza A, Odone A, Aguglia A, Escelsior A, Serafini G. A breakthrough in research on depression screening: from validation to efficacy studies. Acta Biomed. (2021) 92:e2021215. doi: 10.23750/abm.v92i3.11574

38. Soysal P, Veronese N, Thompson T, Kahl KG, Fernandes BS, Prina AM. Relationship between depression and frailty in older adults: a systematic review and meta-analysis. Ageing Res Rev. (2017) 36:7887. doi: 10.1016/j.arr.2017.03.005

39. Chao CT, Wang J, Huang JW, Chan DC, Chien KL. Frailty predicts an increased risk of end-stage renal disease with risk competition by mortality among 165,461 diabetic kidney disease patients. Aging Dis. (2019) 10:1270-81. doi: 10.14336/AD.201 9.0216 
40. Chao CT, Yeh HY, Tsia YT, Chiang CK, Chen HWA. combined microRNA and target protein-based panel for predicting the probability and severity of uraemic vascular calcification: a translational study. Cardiovasc Res. (2021) 117:1958-73. doi: $10.1093 /$ cvr/cvaa255

Conflict of Interest: The authors declare that the research was conducted in the absence of any commercial or financial relationships that could be construed as a potential conflict ofinterest.

Publisher's Note: All claims expressed in this article are solely those of the authors and do not necessarily represent those of their affiliated organizations, or those of the publisher, the editors and the reviewers. Any product that may be evaluated in this article, or claim that may be made by its manufacturer, is not guaranteed or endorsed by the publisher.

Copyright $\odot 2022$ Chi, Lee, Chao and Huang. This is an open-access article distributed under the terms of the Creative Commons Attribution License (CC BY). The use, distribution or reproduction in other forums is permitted, provided the original author(s) and the copyright owner(s) are credited and that the original publication in this journal is cited, in accordance with accepted academic practice. No use, distribution or reproduction is permitted which does not comply with these terms. 